\title{
Validating A Plasma Momentum Flux Sensor Against An Inverted Pendulum Thrust Stand
}

\author{
Benjamin W. Longmier ${ }^{1}$ \\ University of Houston, Department of Physics, Houston, TX 77204, USA \\ Bryan M. Reid ${ }^{2}$, Alec D. Gallimore ${ }^{3}$ \\ University of Michigan, Department of Aerospace Engineering, Ann Arbor, MI 48109 USA \\ Franklin R. Chang-Díaz ${ }^{4}$, Jared P. Squire ${ }^{5}$, Tim W. Glover ${ }^{6}$ \\ Ad Astra Rocket Company, Webster, TX 77598, USA \\ Greg Chavers ${ }^{7}$ \\ NASA Marshall Space Flight Center, Huntsville, AL 35812, USA \\ Edgar A. Bering, III $^{8}$ \\ University of Houston, Departments of Physics and ECE, Houston, TX 77204, USA
}

\begin{abstract}
The accuracy of a plasma impact force sensor was compared to that of the more commonly used inverted pendulum thrust stand using a $5 \mathrm{~kW}$ Xe Hall thruster. A plasma momentum flux sensor (PMFS) was designed and constructed based on a previous NASAMarshall Space Flight Center (MSFC) design. Real-time force measurements were made with both the PMFS and the inverted pendulum thrust stand. The PMFS measured the force exerted onto it from the Hall thruster exhaust plume with a resolution of $0.1 \mathrm{mN}$, and an average accuracy better than $\mathbf{9 8 \%}$ compared to the thrust stand. Experiments were completed at the Large Vacuum Test Facility (LVTF) at the University of Michigan. The total force from the Hall thruster was modulated from $34 \mathrm{mN}$ to $356 \mathrm{mN}$ by varying both the anode voltage, from $150 \mathrm{~V}$ to $500 \mathrm{~V}$, and the neutral Xe gas flow rate from $5 \mathrm{mg} / \mathrm{s}$ to $15 \mathrm{mg} / \mathrm{s}$. The majority of the force data taken during the experiment campaign was completed as a "blind study" where force measurements from both techniques were disclosed only after the experiment was completed.
\end{abstract}

\footnotetext{
${ }^{1}$ Post-Doctoral Fellow, Physics Department, University of Houston, 141 W. Bay Area Blvd., Member

${ }^{2}$ Ph.D. Candidate, Aerospace Engineering, University of Michigan, 1919 Green Rd Room B107, Member

${ }^{3}$ Professor of Aerospace Engineering and of Applied Physics, Associate Dean for Academic Programs and Initiatives, Aerospace Engineering, 1919 Green Rd Room B107, Associate Fellow

${ }^{4}$ Chief Executive Officer, Ad Astra Rocket Company, 141 W. Bay Area Blvd., Associate Fellow

${ }^{5}$ Director of Research, Ad Astra Rocket Company, 141 W. Bay Area Blvd. Member

${ }^{6}$ Director of Development, Ad Astra Rocket Company, 141 W. Bay Area Blvd., Member

${ }^{7}$ Aerospace Engineer, Mission Operations Laboratory, NASA Marshall Space Flight Center, Member

${ }^{8}$ Professor, Physics and ECE, 617 Science \& Research Bldg 1, Associate Fellow
} 


\section{Introduction}

T $\mathrm{T}$ is often physically or financially impractical to measure the force produced by an electric thruster using the traditional technique of mounting the thruster on a pendulum thrust stand. Instead, a simple, robust, and low-cost plasma impact force sensor can be used to reliably determine the total force produced by an electric propulsion thruster. Until now, only a handful ${ }^{1-4}$ of experiments have focused on impact force sensors. Virtually no experiments have attempted to compare the accuracy of an impact thrust sensor to the more commonly used pendulum thrust stand. This experimental campaign, conducted at the Large Vacuum Test Facility (LVTF) at the University of Michigan, demonstrated that the thrust of a $5 \mathrm{~kW}$ Hall thruster inferred from measurements made with a plasma impact force sensor agrees very closely with the thrust measurements made by a conventional pendulum thrust stand. Experimental tests were completed using the P5 Hall thruster, a $5 \mathrm{~kW}$ Xe experimental thruster developed jointly by the University of Michigan and the U.S. Air force Research Laboratory (AFRL) ${ }^{5-7}$

The plasma momentum flux sensor (PMFS) described in this paper was designed and constructed based on a previous NASA Marshall Space Flight Center (MSFC) design. ${ }^{1}$ The PMFS was placed in the flowing plasma stream of a Xe Hall thruster where real time force measurements were made with both the PMFS and the inverted pendulum thrust stand. The PMFS was able to measure the force exerted on it with a resolution of $0.1 \mathrm{mN}$, and an average accuracy better than $98 \%$ compared to the inverted pendulum thrust stand. The total force from the Hall thruster was modulated from $34 \mathrm{mN}$ to $356 \mathrm{mN}$ by varying both the anode voltage, from $150 \mathrm{~V}$ to $500 \mathrm{~V}$, and the neutral Xe gas flow rate from $5 \mathrm{mg} / \mathrm{s}$ to $15 \mathrm{mg} / \mathrm{s}$. Additionally, the majority of the force data taken during the experiment campaign was completed as a 'blind study' where force measurements from both techniques were disclosed only after the experiment was completed. Though the experiments show a high accuracy for Hall thrusters with ion energies ranging from $130 \mathrm{eV}$ to $440 \mathrm{eV}$, the PMFS showed no indication of saturation with ion energy, plasma flux, local magnetic field strength, or force magnitude, indicating that the full range of the PMFS usefulness is still largely unexplored. The PMFS may find uses in many flowing plasma and electric propulsion applications where an accurate, sub- $\$ 500$ force sensor is required.

\section{Experimental Setup}

\section{A. Concept and Construction of the PMFS}

The PMFS was developed and constructed based on a previous NASA-Marshall Space Flight Center design. ${ }^{1}$ The PMFS consists of a 9-centimeter-diameter graphite target disc attached to a 10-centimeter-long insulating alumina rod. The stiff alumina rod then connects to a small titanium bar $(5.72 \mathrm{~cm} \mathrm{x} 1.30 \mathrm{~cm})$ where a series of 4 high output semiconductor strain gauges are mounted between two holes on an 'isthmus' on the titanium bar, as seen in Fig. 1. The isthmus acts as a stress concentrator and increases the sensitivity of the device. The strain gauges are connected electrically in a Wheatstone bridge configuration so that changes in temperature of the titanium bar do not affect the linearity of the strain gauge output. When the graphite disc is immersed in flowing plasma (e.g. the plume of a Hall thruster) the force from the plasma impacting the graphite target is translated into a strain in the titanium beam through a moment arm equal to the length of the alumina rod plus the clamp length. A small graphite shield was also used to keep the entire titanium bar and strain gauge assembly shielded from the flowing plasma, and associated thermal and electrical noise.

The resolution of the PMFS and the inverted pendulum thrust stand described in this paper was $0.1 \mathrm{mN}$ and 0.5 $\mathrm{mN}$ respectively, which allowed for sufficiently sensitive measurements of the force applied by the exhaust plasma. An average discrepancy between the measured force from the PMFS and the measured force from the University of Michigan thrust stand of approximately $2 \%$ was observed, indicating a good agreement between the two force measurement techniques. When the Hall thruster power level was throttled from $0.71 \mathrm{~kW}$ to $7.65 \mathrm{~kW}$, the largest discrepancy between the two force measurement methods was 5.7\%, which occurred at a power level of $3.15 \mathrm{~kW}$.

For reference, the typical error associated with the inverted pendulum thrust stand is $\pm 2 \mathrm{mN}$ for a measured force of $100 \mathrm{mN}$, indicating that the typical $2 \%$ difference observed between the two force measurement techniques is usually within the error associated with the thrust stand. 


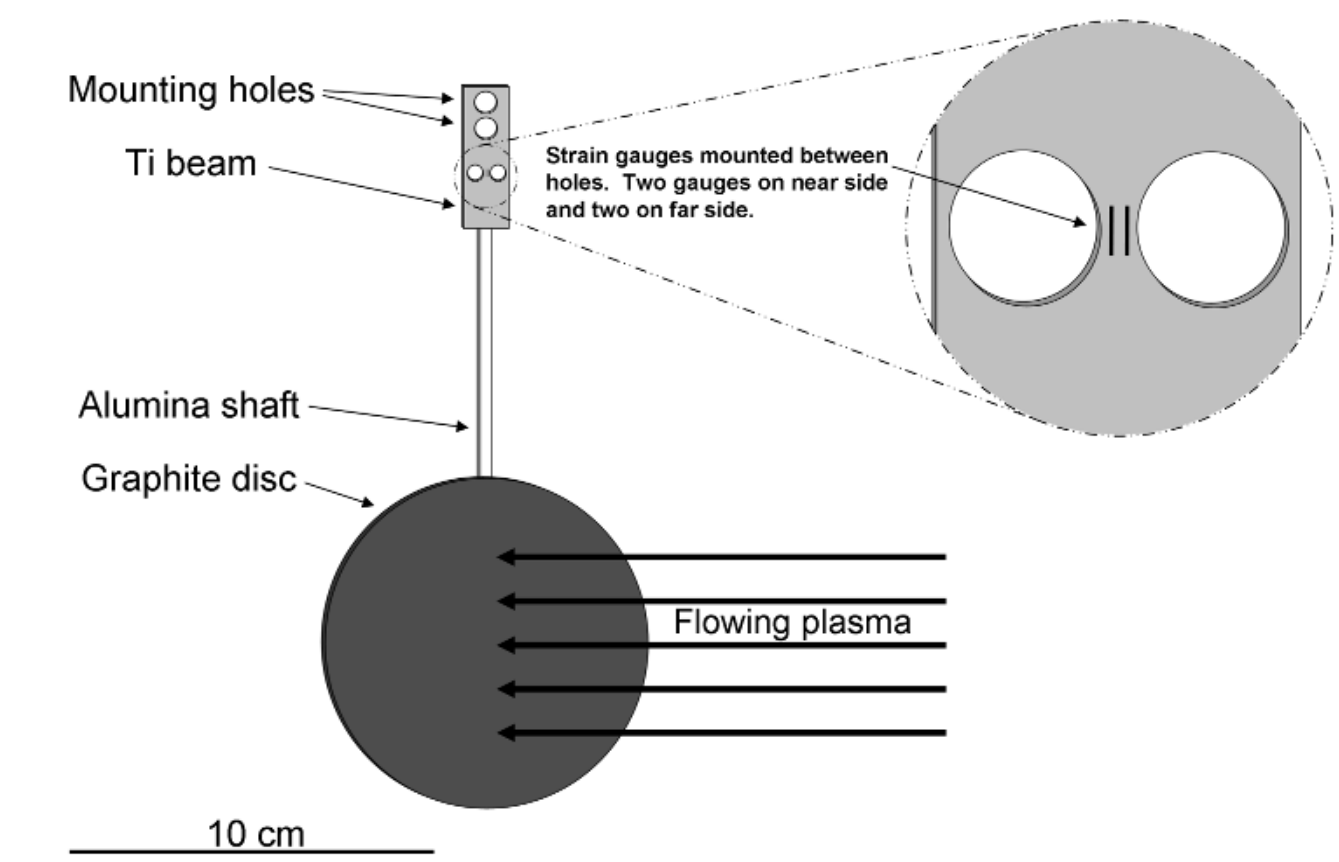

a)

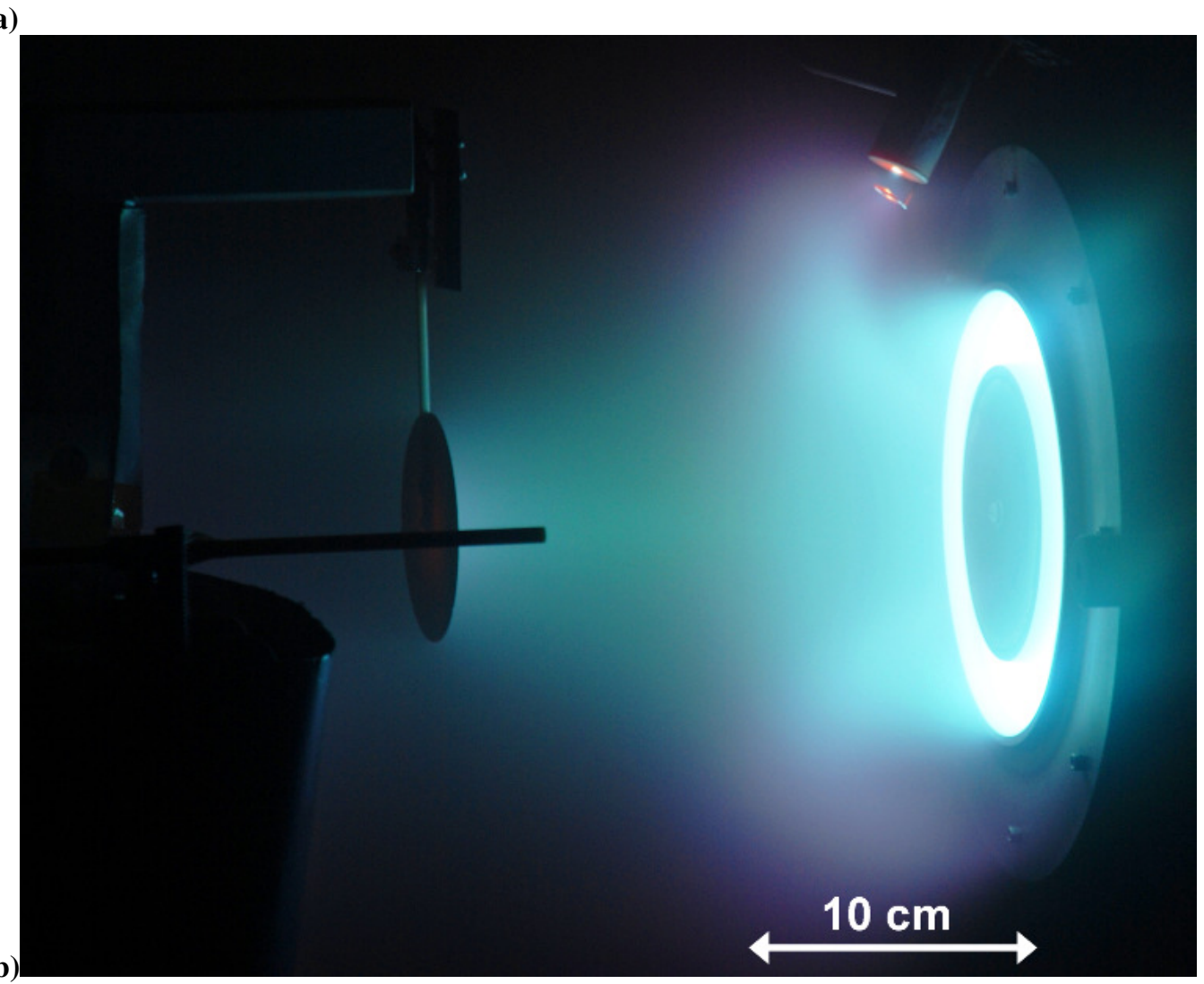

Figure 1. Schematic of the PMFS assembly and zoom in of strain gauge arrangement mounted on the Ti isthmus, a), and a photo of the P-5 Hall thruster and PMFS during the closest approach. The Faraday probe biased into ion saturation can be seen in the foreground and the PMFS graphite target disc in the background in Fig. 1b). 
If an increased force resolution were required, the length of the alumina moment arm could be increased, acting to increase the output from the strain gauges for a particular force applied to the graphite target. However, increasing the arm length of the device also decreases the resonant frequency response. This limitation is generally not a concern for steady-state thruster operation. If the thruster (or some other source of flowing plasma) were operated in a pulsed mode, then data analysis is simplified if the moment arm was selected such that the natural period of the PMFS device is much shorter than the thruster pulse duration.

The diameter of the graphite target used in this experiment campaign was smaller than the diameter of the Hall thruster plume, therefore the target only measured a portion of the total force generated by the Hall thruster in each measurement. The PMFS target diameter was 50\% of the P-5 thruster channel O.D. An azimuthally integrated radial profile of the ion flux was used to account for the portion of the plasma plume that was not intercepted by the graphite target. For each force measurement presented in this paper, a corresponding radial profile of the ion flux was collected and used to determine the total force produced by the thruster. Figure 2 is a representative radial ion flux profile from the P5 Hall thruster plume; in this case, the graphite target was $50 \mathrm{~cm}$ downstream from the exit plane of the thruster.

The ratio of the total ion flux $(\mathrm{r}=0$ to $\mathrm{r}=100 \mathrm{~cm})$, numerically integrated over the entire plume assuming cylindrical symmetry, to that of the ion flux intercepted by the graphite target $(\mathrm{r}=0 \mathrm{~cm}$ to $\mathrm{r}=9 \mathrm{~cm})$ is given by

$$
\frac{\sum_{x=0}^{x=1000} \pi\left(r_{\mathrm{x}+1}^{2}-r_{\mathrm{x}}^{2}\right) I\left(r_{\mathrm{x}}\right)}{\sum_{x=0}^{x=90} \pi\left(r_{\mathrm{x}+1}^{2}-r_{\mathrm{x}}^{2}\right) I\left(r_{\mathrm{x}}\right)}
$$

where $I\left(r_{\mathrm{x}}\right)$ is the ion current as measured by a Faraday probe biased into ion saturation at a radius $r_{\mathrm{x}}$ in the plasma exhaust. Here, $x$ ranges from 0 to 1,000 for $r_{\mathrm{x}}$ values from 0 to $100 \mathrm{~cm}$.

The total force, $F_{\text {Total }}$, produced by the Hall thruster is determined by multiplying the force measured by the graphite target, $F_{\text {Target }}$, by Eqn. (1), which becomes

$$
F_{\text {Total }}=F_{\text {Target }} \frac{\sum_{x=0}^{x=1000} \pi\left(r_{\mathrm{x}+1}^{2}-r_{\mathrm{x}}^{2}\right) I\left(r_{\mathrm{x}}\right)}{\sum_{x=0}^{x=90} \pi\left(r_{\mathrm{x}+1}^{2}-r_{\mathrm{x}}^{2}\right) I\left(r_{\mathrm{x}}\right)}
$$

Charge-exchange (CEX) particles and doubly-charged ions do not affect the accuracy of the PMFS as long as the fraction of these CEX neutrals and doubly-charged ions is small compared to singly-charged ions, or the CEX and doubly-ionized fluxes are directly proportional to the ion flux. This is a reasonable assumption based on previous data taken with the P5 Hall Thruster. ${ }^{9}$

The assumption that the thruster plume is symmetric in the azimuthal direction leads to the largest source of error with the PMFS device. One way to reduce this error is to construct a 2-D map of the ion flux profile; however, this mapping was not performed. In this series of experiments it was found that assuming azimuthal symmetry led 
to no larger than a $5.7 \%$ difference between the force measured by the PMFS and the inverted pendulum thrust stand, and typically resulted in no more than a $2 \%$ difference.

Once a total force measurement was numerically integrated from the PMFS measurement and ion flux profile, momentum reflection and sputtering were taken into consideration and corrections were made, as described in section IV A. The energy and mass of the incident Xe ions was also large compared to the surface binding energy and mass of the carbon atoms within the graphite target. Simulations from the Stopping and Range of Ions in Matter (SRIM) code ${ }^{10}$ show that the Xe ions implant themselves several microns into the surface of the graphite target and do not immediately 'bounce' off of the target surface. This ion implantation claim is supported by the experimental force comparison results in sections III A and C. The Xe ions that become implanted within the graphite target may eventually make their way back to the front surface and escape from the target. However, the neutral Xe atoms would escape in an isotropic distribution as cold neutrals and would carry away negligible momentum compared to the incident $\mathrm{Xe}$ ions. The momentum that sputtered $\mathrm{C}$ atoms carry away with them is at most $1 \%$ of the incident $\mathrm{Xe}$ ion momentum and amounts to a small correction to the measured force, see section IV A for calculations.

\section{B. Calibration}

After the PMFS was installed in a rigid location within the University of Michigan vacuum chamber, in this case on a 2-axis translation stage, and before the vacuum pump down, the PMFS was calibrated in order to find an accurate relationship between the output of the strain gauges and the force applied to the target. Calibration was performed in the same manner as was previously described by Chavers et al. ${ }^{1}$, in which a set of calibrated weights were hung from a tethered string assembly to apply a known tension force on the graphite target disc. Figure 3 shows a representative example of the force-to-voltage relation for the particular device used in this experiment campaign. The strain sensors had an output voltage-to-force relation of $0.64 \pm 0.01 \mathrm{mV} / \mathrm{mN}$ (or $1.56 \mathrm{mN} / \mathrm{mV}$ ). If the PMFS was correctly leveled within the vacuum chamber prior to testing, the resulting force per unit voltage line would intercept the origin, as depicted in Fig. 3.

The data from Fig. 3 also indicate that the strain gauges are linear over a force range from 0 to at least $50 \mathrm{mN}$ for this particular device. Because the graphite target did not intercept all of the Hall thruster exhaust plume, this translates into a useful measurable Hall thruster force range of at least 0 to $1000 \mathrm{mN}$. A PMFS calibration was performed before and after every vacuum chamber pump down and venting in order to verify that the strain gauges did not exhibit a drift or creep in time. During the PMFS Hall thruster experiments, both pre- and post-strain gauge calibrations agreed to within $0.3 \%$.

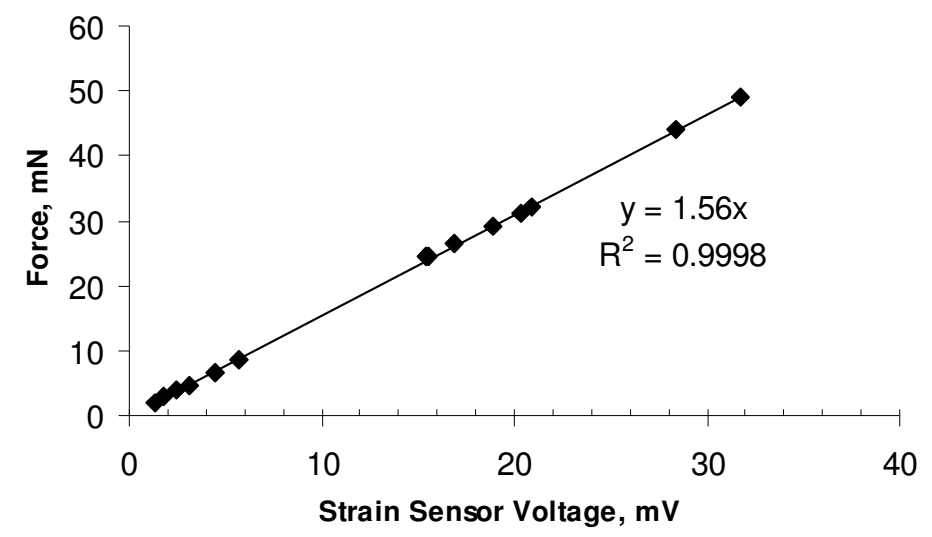

Figure 3. A calibration graph is used to convert strain sensor voltage to an applied force on the target.

\section{Hall Thruster}

All data within this paper, with the exception of Section III D, were taken using the P5 Xe Hall Thruster, a 5-kW Hall thruster designed and used primarily for research purposes at the University of Michigan and the Air Force Research Laboratory at Edwards Air Force Base. Previous data indicate that the P5 has performance characteristics comparable to commercial space-qualified 5-kW Hall thrusters. ${ }^{5-8}$ The P5 has a discharge chamber outer diameter of $173 \mathrm{~mm}$ and channel width of $25 \mathrm{~mm}$. Although designed for steady-state operation at $5 \mathrm{~kW}$, the thruster was operated at power levels from $0.71 \mathrm{~kW}(150 \mathrm{~V}, 4.70 \mathrm{~A})$ to $7.65 \mathrm{~kW}(500 \mathrm{~V}, 15.30 \mathrm{~A})$ during this experiment. The P5 used only xenon gas as the propellant for the duration of this testing campaign and for all data presented in this paper.

\section{Chamber and diagnostic setup}

The LVTF, a cylindrical stainless-steel clad vacuum chamber at the University of Michigan-Ann Arbor, is a 9meter-long by 6-meter-diameter chamber used primarily for the testing of research and space qualified electric propulsion thrusters. ${ }^{11}$ The chamber is evacuated by two 2,000 cfm blowers and four $400 \mathrm{cfm}$ mechanical pumps to a rough vacuum level of 30 to 100 mTorr. ${ }^{11}$ To reach high vacuum, $\sim 10^{-7}$ Torr, the vacuum chamber employs seven 
CVI TM-1200 nude cryopumps, with a combined pumping speed of 500,000 1/s of air, and 240,000 1/s of xenon. However, because of the low flow rates of the P5 thruster, only 4 of the 7 cryopumps were used during this experiment campaign with a resulting Xe pumping speed of 140,000 1/s. The 4 cryopumps yielded an average base vacuum pressure of $3 \times 10^{-7}$ Torr, and an uncorrected (indicated) chamber pressure of $7 \times 10^{-6}$ Torr with a combined thruster and cathode flow rate of $5 \mathrm{mg} / \mathrm{s} \mathrm{Xe}$.

The P5 thruster was mounted on an inverted pendulum thrust stand, described in detail by Haag et al. ${ }^{12}$ The inverted pendulum thrust stand, and hence the P5 thruster, was fixed in place within the vacuum chamber, and the PMFS was mounted on a 2-axis translation stage within the vacuum chamber. The translation stage allowed the PMFS to scan a separation distance of 94 to $23 \mathrm{~cm}$ from the thruster exit plane, and allowed for a lateral range of $100 \mathrm{~cm}: 70 \mathrm{~cm}$ from the axis in one direction and $30 \mathrm{~cm}$ from the axis in the other direction. For all force measurements the graphite PMFS target was centered radially and placed at various axial separation distances. However, the translation stage moved through its entire lateral range of motion in order to obtain a radial ion flux profile with a Faraday probe biased into ion saturation. A 3-millimeter-diameter tungsten rod enclosed in an alumina shroud served as the Faraday probe. The tip of the rod was flush with the alumina tube in order to help prevent a radial sheath expansion with biases more negative than the floating potential. The Faraday probe was mounted at the same vertical and axial position as the graphite PMFS target, but with a $20 \mathrm{~cm}$ radial offset from the PMFS target. The surface of the Faraday probe was also cleaned for 10 minutes via xenon ion bombardment prior to data collection.

In order to measure the ion energy in the plume, a Retarding Potential Analyzer (RPA) was installed on a 60centimeter-long boom that was able to sweep through the plume. ${ }^{13}$ Measurements were then taken with the RPA located $58.5 \mathrm{~cm}$ from the exit plane of the thruster. The RPA was mounted on the boom structure so that it could be moved out of the thruster plume when PMFS measurements were taken. A small floating probe was also installed on the boom and was used to measure the plasma floating potential $58.5 \mathrm{~cm}$ from the exit plane of the thruster.

\section{Experimental Demonstration}

\section{A. Thruster-Sensor Separation Distance}

An initial thruster-to-target separation distance experiment was performed in order to determine which separation distances could be tolerated without significantly altering the performance of the thruster. The separation distance was decreased from $94 \mathrm{~cm}$ to $23 \mathrm{~cm}$ in roughly 10-centimeter increments. For each thrust measurement, the thruster was turned on and allowed to stabilize. Then, a radial ion flux profile was taken with the Faraday probe and a relative thrust measurement was taken with both techniques. The thruster was then turned off, and a baseline thrust measurement was taken, also with both techniques. The difference in signal strength between the thrust-on and thrust-off values was used to determine a thruster force. The slope from the respective PMFS and thrust stand calibration curves (i.e. $\mathrm{mN} / \mathrm{mV}$ ) was then used to determine an absolute force value from the relative force measurements. In this way, simultaneous force measurements could be made with both techniques. This procedure also helped to compensate for any drift in thruster performance or force over time.

An increase in the measured force was registered with both techniques when the PMFS disc approached the Hall thruster as seen in Fig 4. The PMFS data presented in Fig. 4 and Table 1 also include corrections for sputtering based on SRIM/TRIM models ${ }^{10}$ (see Section IV A for further sputtering correction details).

\begin{tabular}{lcccccccc}
\hline Table 1. Measured thrust versus the separation distance of the PMFS target from \\
& \multicolumn{7}{c}{ the Hall thruster. } \\
\hline Separation distance, cm & 94 & 80 & 70 & 60 & 50 & 40 & 30 & 23 \\
PMFS, mN & $\mathbf{7 6 . 1}$ & $\mathbf{7 6 . 6}$ & $\mathbf{7 6 . 8}$ & $\mathbf{7 6 . 8}$ & $\mathbf{7 6 . 9}$ & $\mathbf{7 6 . 9}$ & $\mathbf{7 7 . 1}$ & $\mathbf{7 7 . 3}$ \\
Thrust stand, mN & $\mathbf{7 6 . 3}$ & $\mathbf{7 7 . 1}$ & $\mathbf{7 7 . 6}$ & $\mathbf{7 7 . 9}$ & $\mathbf{7 7 . 9}$ & $\mathbf{7 8 . 4}$ & $\mathbf{7 9 . 2}$ & $\mathbf{7 9 . 2}$ \\
Difference, \% & 0.3 & 0.6 & 1.1 & 1.4 & 1.2 & 1.9 & 2.6 & 2.4 \\
Anode Voltage, V & 300 & 300 & 300 & 300 & 300 & 300 & 300 & 300 \\
Xe flow rate, mg/s & 5 & 5 & 5 & 5 & 5 & 5 & 5 & 5 \\
Anode current, A & 5.1 & 5.1 & 5.1 & 5.1 & 5.1 & 5.1 & 5.1 & 5.1 \\
\hline
\end{tabular}




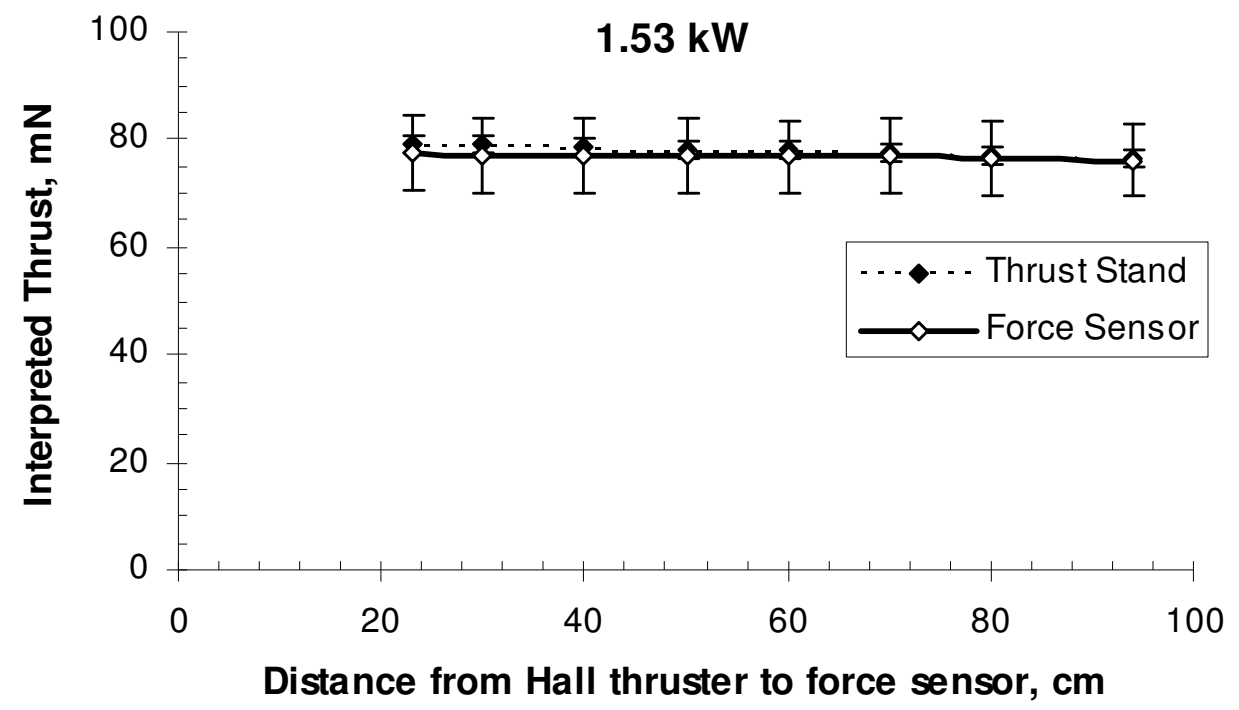

Figure 4. Measured thrust versus the separation distance of the PMFS target and the Hall thruster. The thruster was operated at $1.53 \mathrm{~kW}$, using $5 \mathrm{mg} / \mathrm{s} \mathrm{Xe,} 5.1 \mathrm{~A}$ at $300 \mathrm{~V}$.

The increase in thruster force with reduced thruster-target separation distance was previously observed by Chavers et al. ${ }^{1}$ in a similar experiment (see Section D), and is attributed to an increased neutral pressure near the Hall thruster exit plane. Due to finite facility pressure, background neutrals continually reach the thruster discharge channel, become ionized, accelerated, and artificially increase thrust. ${ }^{14}$ As the PMFS approaches the thruster exit plane, the neutral ingestion is expected to increase because of two effects. First, a fraction of the non-ionized neutrals from the thruster will be reflected from the paddle surface with an isotropic distribution. Second, ions will impact the paddle and recombine at the surface to produce an additional isotropic reflected neutral flux. A fraction of these neutralized ions will also reach the thruster and artificially increase the thrust produced by the thruster. The Hall thruster was operated at $1.53 \mathrm{~kW}$, producing $5.1 \mathrm{~A}$ of discharge current with an anode bias of $300 \mathrm{~V}$, using a neutral Xe flow rate of $5 \mathrm{mg} / \mathrm{s}$. The average difference between the force measurements for the inverted pendulum thrust stand and PMFS techniques is $1.4 \%$, which is considerably less than the error associated with either the PMFS or the inverted pendulum thrust stand for this set of experiments.

\section{B. Blind Study}

The following data from the thrust stand and the PMFS were taken and analyzed as a "blind study." That is, the forces measured by the thrust stand and the PMFS were recorded separately and were only disclosed to each respective research team three weeks later, after a complete calibration and sputter analysis had been performed. The PMFS was positioned on thruster centerline $50 \mathrm{~cm}$ from the exit plane of the thruster while the Xe flow rate and the thruster anode voltage were altered in order to achieve a range of ion energy, ion flux, and total force. Figure 5 shows the thrust from the thrust stand and the PMFS measurements as a function of the calculated Hall thruster power. Table 2 shows the numeric results displayed in Fig. 5.

\begin{tabular}{lccccccc}
\hline \hline Table 2. Measured thrust for a variety of P5 Hall thruster power levels. \\
The PMFS was located 50 cm downstream from the thruster exit plane. \\
\hline Thruster power, kW & 0.71 & 1.50 & 1.58 & 2.49 & 3.15 & 4.38 & 7.65 \\
PMFS, mN & $\mathbf{4 1 . 4}$ & $\mathbf{7 2 . 7}$ & $\mathbf{8 9 . 2}$ & $\mathbf{1 3 8 . 2}$ & $\mathbf{1 4 9 . 7}$ & $\mathbf{1 9 3 . 0}$ & $\mathbf{2 8 4 . 3}$ \\
Thrust stand, mN & $\mathbf{3 3 . 9}$ & $\mathbf{7 3 . 6}$ & $\mathbf{8 5 . 9}$ & $\mathbf{1 3 6 . 6}$ & $\mathbf{1 5 8 . 8}$ & $\mathbf{2 3 7 . 1}$ & $\mathbf{3 5 5 . 8}$ \\
Difference, \% & 22.3 & 1.2 & 3.8 & 1.2 & 5.7 & 18.6 & 20.1 \\
Anode Voltage, V & 150 & 300 & 150 & 150 & 300 & 300 & 500 \\
Xe flow rate, mg/s & 5 & 5 & 10 & 15 & 10 & 15 & 15 \\
Anode current, A & 4.7 & 5.0 & 10.5 & 16.6 & 10.5 & 14.6 & 15.3 \\
\hline
\end{tabular}




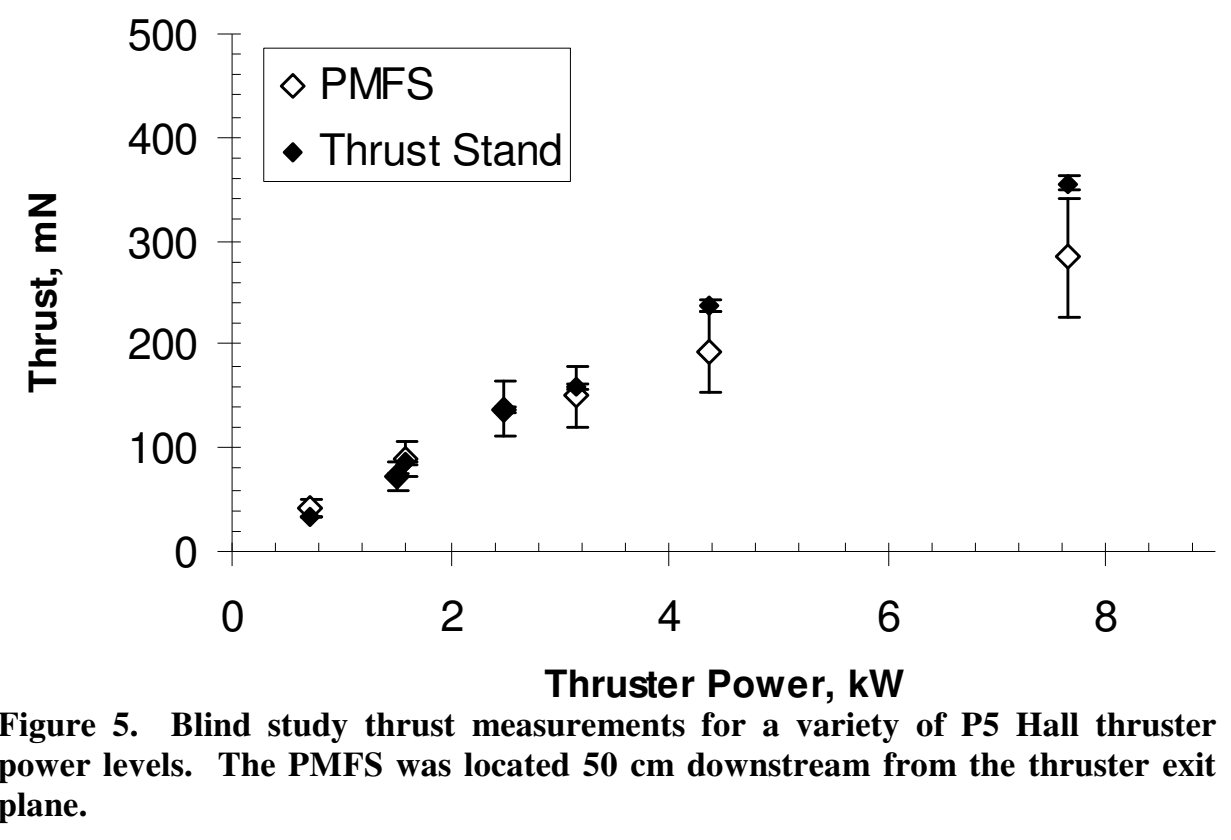

The large discrepancies associated with the $7.65-\mathrm{kW}, 4.38-\mathrm{kW}$, and $0.71-\mathrm{kW}$ power levels were later determined to be due to an incorrect scaling value used with the radial ion flux traces for the PMFS. This issue was corrected after the blind study and the corrected data are presented in Section III C.

\section{Corrections to Blind Study}

After both sets of force data from the blind study were exchanged between research teams at the University of Michigan and the University of Houston, it was discovered that three of the PMFS data points fell much farther away from the inverted pendulum data points. In a post "blind study" analysis, it was discovered that an incorrect center-line was used for three of the radial ion flux traces, throwing off the total force value for the three PMFS measurements with a larger discrepancy. Figure 6 shows a graph of the radial ion flux profiles for several thruster gas flow rates and anode voltage levels with the correct thruster centerline superimposed on the graph.

During the initial data analysis, an incorrect plume centerline was chosen based on the two largest peaks and the smallest peak in Fig. 6, instead of the true plume centerline. This incorrect centerline was used to calculate the force values for the two largest peaks and the smallest peak, resulting in three incorrect force values. The remaining ion flux peaks were analyzed separately with the correct thruster centerline. The clearer bimodal nature of the remaining peaks lead to the correct choice of the thruster plume centerline and the correct calculation of force values for the PMFS. The updated and correct results are shown in Fig. 7 and Table 3. A more accurate centerline for all of the peaks, found by fitting a bimodal distribution to the data in Fig. 6, is located at a radial position of 133.5 $\mathrm{cm}$ and shown in Fig. 6. The Faraday probe that was mounted on the translation stage had a radial range from 0 to $138.1 \mathrm{~cm}$, where the thruster plume centerline located at $133.5 \mathrm{~cm}$.

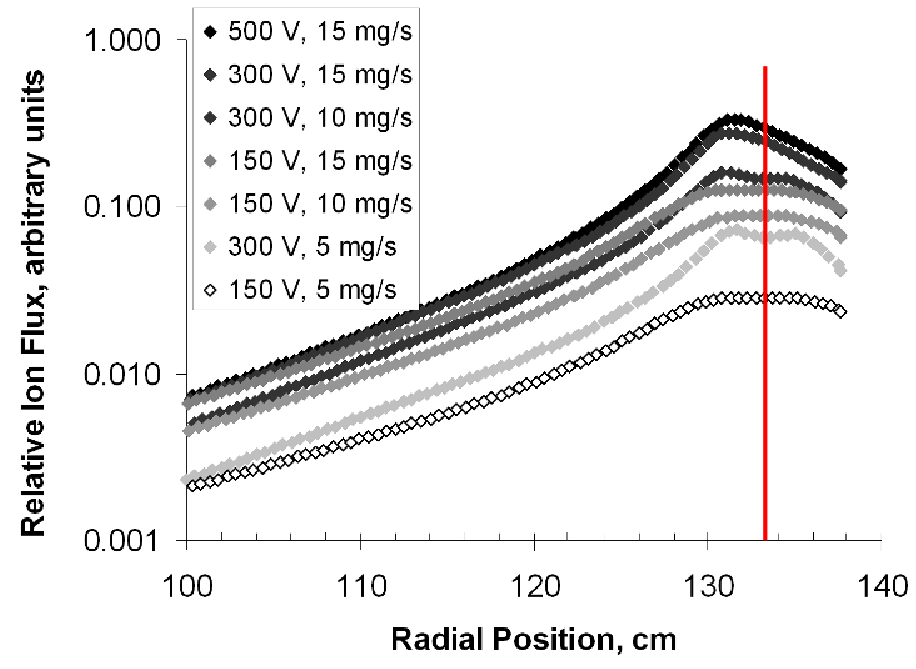

Figure 6. A graph of the relative radial ion flux profiles for several thruster gas flow rates and anode voltage levels. The PMFS and the Faraday probe were located $50 \mathrm{~cm}$ downstream from the thruster exit plane. The solid vertical red line shows the correct plume centerline. 


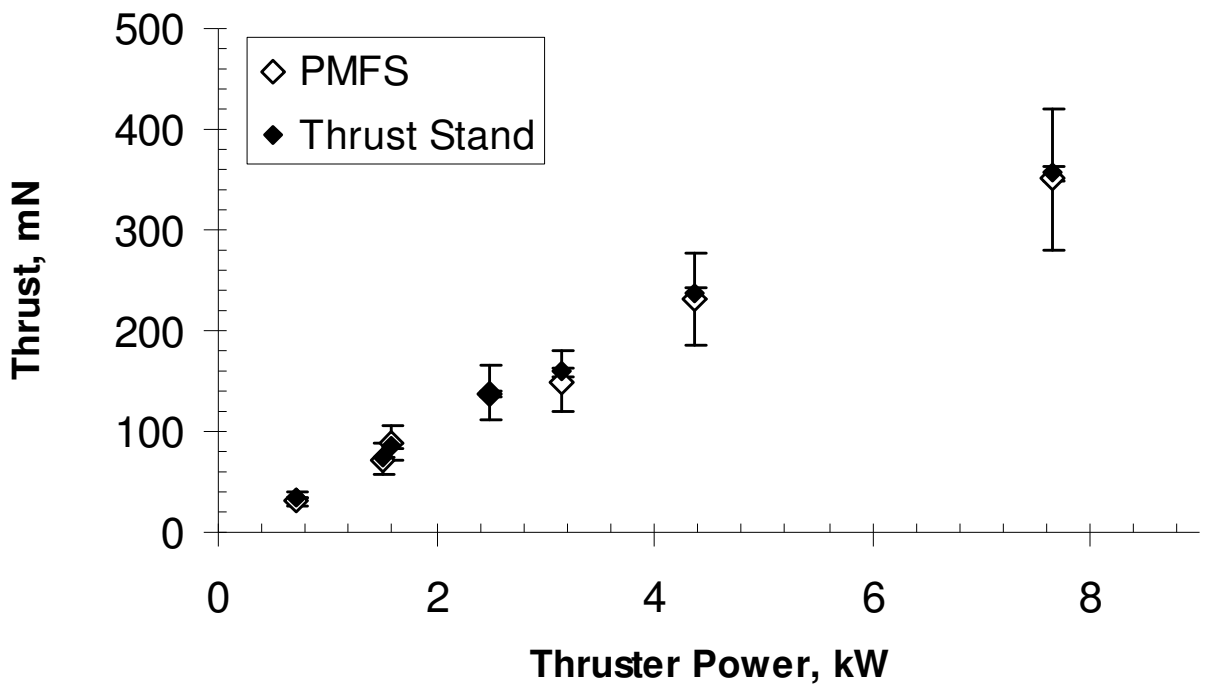

Figure 7. Corrected blind study thrust measurements for a variety of P5 Hall thruster power levels. The PMFS was located $50 \mathrm{~cm}$ downstream from the thruster exit plane.

Table 3. Measured thrust for a variety of $P 5$ Hall thruster power levels. The PMFS was located $50 \mathrm{~cm}$ downstream from the thruster exit plane.

\begin{tabular}{lccccccc} 
Thruster power, kW & 0.71 & 1.50 & 1.58 & 2.49 & 3.15 & 4.38 & 7.65 \\
PMFS, mN & $\mathbf{3 2 . 6}$ & $\mathbf{7 2 . 7}$ & $\mathbf{8 9 . 2}$ & $\mathbf{1 3 8 . 2}$ & $\mathbf{1 4 9 . 7}$ & $\mathbf{2 3 1 . 4}$ & $\mathbf{3 5 0 . 7}$ \\
Thrust stand, mN & $\mathbf{3 3 . 9}$ & $\mathbf{7 3 . 6}$ & $\mathbf{8 5 . 9}$ & $\mathbf{1 3 6 . 6}$ & $\mathbf{1 5 8 . 8}$ & $\mathbf{2 3 7 . 1}$ & $\mathbf{3 5 5 . 8}$ \\
Difference, \% & 3.6 & 1.2 & 3.8 & 1.2 & 5.7 & 2.4 & 1.4 \\
Anode Voltage, V & 150 & 300 & 150 & 150 & 300 & 300 & 500 \\
Xe flow rate, mg/s & 5 & 5 & 10 & 15 & 10 & 15 & 15 \\
Anode current, A & 4.7 & 5.0 & 10.5 & 16.6 & 10.5 & 14.6 & 15.3 \\
\hline
\end{tabular}


After the correct centerline was used to calculate the total thrust with the PMFS technique, the three previously errant force measurements agreed much more closely with the inverted pendulum thrust values. After using the correct plume centerline, the largest difference between the thrust stand measurements and the PMFS measurements was $5.7 \%$, with an average discrepancy of $2.8 \%$, as seen in Table 3 .

In an effort to identify a trend in the PMFS accuracy as a function of ion energy and neutral gas flow rate, the relevant PMFS and thrust stand force data are graphed as a function of the thruster neutral flow rate, Fig. 8, and as a function of the thruster anode voltage, Fig. 9. No clear correlation of PMFS accuracy is obvious as the anode voltage or neutral gas flow rate are varied. To first order, the discrepancy between the PMFS and the thrust stand appears to be largely unaffected by the Hall thruster system parameters. However, data from Fig. 4 does indicate a discernable trend, with a larger discrepancy between the two force measurement techniques as the PMFS approaches the thruster exit plane from a separation distance of $94 \mathrm{~cm}$ to $23 \mathrm{~cm}$.

The PMFS has proven to be accurate compared to the inverted pendulum thrust stand with a maximum observed difference of 5.7\% over a large range of applied force, ion energy, and neutral gas flow settings. The PMFS should find considerable application in the low-thrust plasma community due to its low cost, robustness, and verified accuracy.

\section{Momentum Flux Parameters}

\section{A. Surface Sputtering}

Target material choice for the PMFS is a nontrivial task owing to the bombardment from high energy ions in the exhaust plume of the Hall thruster. At a maximum operating power level, the P5 Hall thruster was able to produce incident ions with an energy of approximately $440 \mathrm{eV}$. This ion energy is far beyond the sputter threshold of any material. One simply tries to reduce the sputtering yield in order to reduce the associated momentum corrections and to reduce contamination of the Hall Thruster from sputtered target material. With an $\mathrm{Ar}, \mathrm{Kr}$, or $\mathrm{Xe}$ propellant, the natural target material choice for low sputter yields is graphite. This low yield is in part a result of the covalent $\mathrm{C}$ $\mathrm{C}$ bond strength which results in the lowest sputter yields compared to any other material (with the exception of diamond) when the incident particles are more than twice as massive as the target atom. ${ }^{15}$ Experiments have also shown that for incident Xe ions, graphite has the lowest sputtering yield (with the exception of diamond). ${ }^{16}$ In order to quantify sputtering yield rates in this experiment campaign, an RPA was used to measure the incident ion energy from the Hall thruster for every force measurement data point. For typical operation, the P5 Hall thruster produces ions with an energy equal to $50 \mathrm{~V}$ less than the Anode voltage. That is, $250 \mathrm{eV} \mathrm{Xe}$ ions are observed from an anode setting of $300 \mathrm{~V}$ with the P5 Hall thruster. ${ }^{13}$

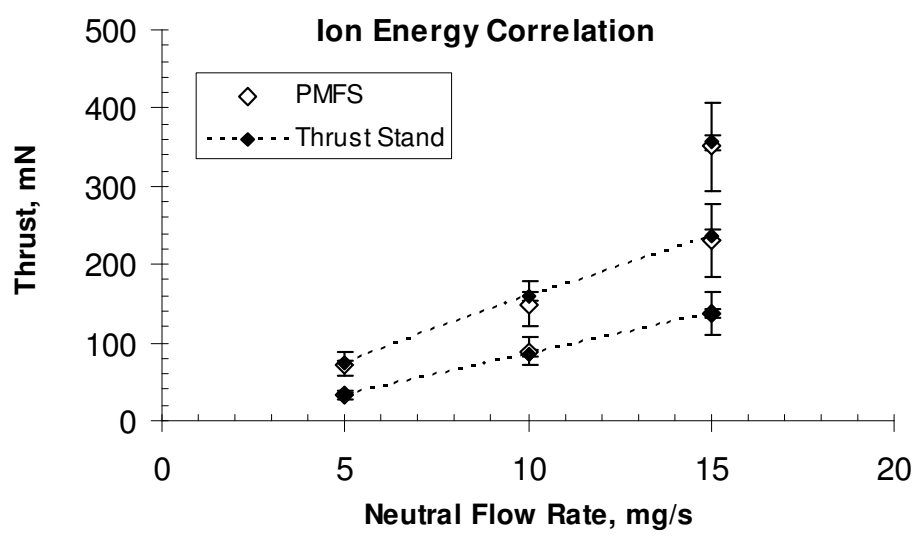

Figure 8. Corrected blind study thrust measurements for a variety of $P 5$ Hall thruster power levels. The PMFS was located $50 \mathrm{~cm}$ downstream from the thruster exit plane.

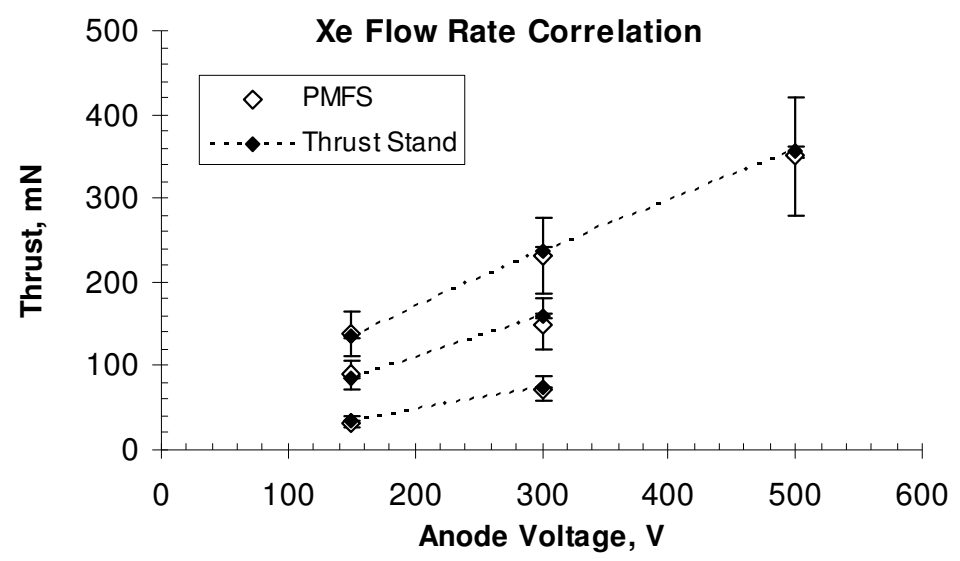

Figure 9. Corrected blind study thrust measurements for a variety of P5 Hall thruster power levels. The PMFS was located at $50 \mathrm{~cm}$ downstream from the thruster exit plane. 
The sputtering yield from a target surface is also highly dependent on the incident angle of the incoming particle, where incident particle trajectories that are normal to the surface generally produce lower sputtering yields and incident particle trajectories that are $\sim 70$ to 85 degrees from normal have the largest sputtering yields. Figure $10 a$ ) and b) show the simulated ${ }^{10}$ graphite sputter yield and energy per sputtered carbon atom as a function of incident angle from normal for various incident Xe ion energies. In Fig. 10, a Xe particle with an incident angle of 0 degrees means the particle is perpendicular (normal) to the surface.

SRIM sputter yield and sputtered particle energy simulations were completed once the incident $\mathrm{Xe}$ ion energy was determined from RPA data and the possible incident ion trajectories were determined from the target to thruster separation distance and thruster dimensions. The SRIM simulations gave the yield and energy of sputtered $\mathrm{C}$ atoms. These results were used to find the added momentum flux on the target caused by the exiting sputtered particles. The sputter simulation output is presented in Tables 4 and 5 for the separation distance experiment and the corrected "blind study" experiment respectively. The last row in Tables 4 and 5 shows the momentum change on the PMFS force target that was caused by sputtered $\mathrm{C}$ atoms leaving the target surface.
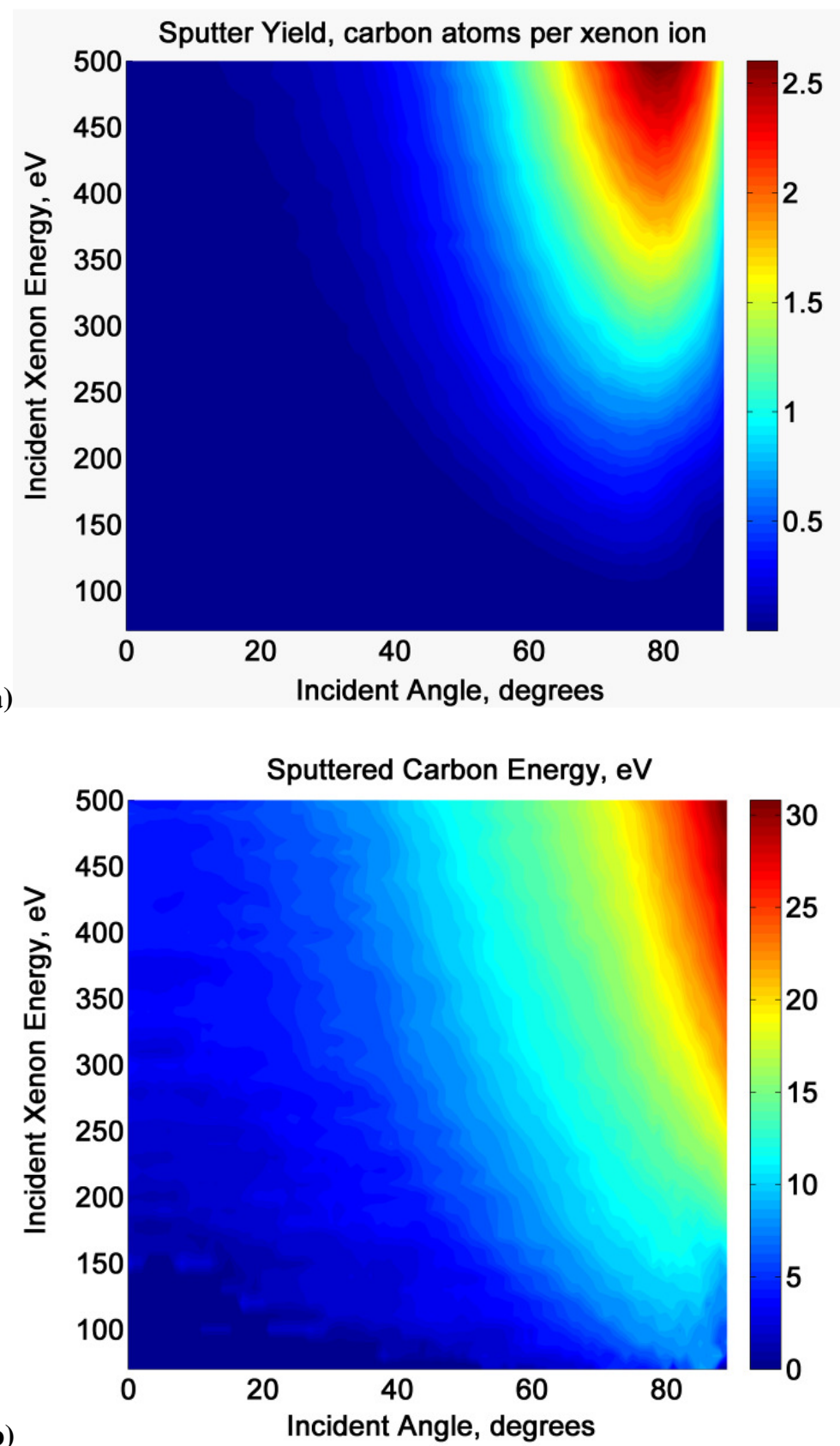

Figure 10. Carbon sputter yield ( $\mathrm{C}$ atom released per incident $\mathrm{Xe}$ ion) a), and average sputtered $C$ atom energy b), as a function of incident angle from the target perpendicular for various incident $\mathrm{Xe}$ ion energies. 


\begin{tabular}{|c|c|c|c|c|c|c|c|c|}
\hline Separation distance, $\mathrm{cm}$ & 94 & 80 & 70 & 60 & 50 & 40 & 30 & 23 \\
\hline PMFS, mN & 76.1 & 76.6 & 76.8 & 76.8 & 76.9 & 76.9 & 77.1 & 77.3 \\
\hline Thrust stand, $\mathrm{mN}$ & 76.3 & 77.1 & 77.6 & 77.9 & 77.9 & 78.4 & 79.2 & 79.2 \\
\hline Difference, \% & 0.3 & 0.6 & 1.1 & 1.4 & 1.2 & 1.9 & 2.6 & 2.4 \\
\hline Anode voltage, $\mathrm{V}$ & 300 & 300 & 300 & 300 & 300 & 300 & 300 & 300 \\
\hline $\mathrm{Xe}$ ion energy, eV & 250 & 250 & 250 & 250 & 250 & 250 & 250 & 250 \\
\hline Integrated sputter yield, atoms/ion & 0.21 & 0.22 & 0.22 & 0.23 & 0.25 & 0.28 & 0.30 & 0.40 \\
\hline Sputtered energy of carbon, eV & 2.0 & 2.1 & 2.2 & 2.2 & 2.5 & 2.5 & 2.7 & 3.1 \\
\hline Momentum increase, \% & 0.6 & 0.6 & 0.6 & 0.7 & 0.8 & 0.8 & 0.9 & 1.3 \\
\hline
\end{tabular}

Table 5. Carbon sputter yield, average sputtered $\mathrm{C}$ atom energy, and the associated momentum increase as a function of incident $\mathrm{Xe}$ angle and energy for various thruster anode and gas flow settings.

\begin{tabular}{lccccccc}
\hline Thruster power, $\mathrm{kW}$ & 0.71 & 1.50 & 1.58 & 2.49 & 3.15 & 4.38 & 7.65 \\
PMFS, mN & 32.6 & 72.7 & 89.2 & 138.2 & 149.7 & 231.4 & 350.7 \\
Thrust stand, mN & 33.9 & 73.6 & 85.9 & 136.6 & 158.8 & 237.1 & 355.8 \\
Difference, \% & 3.6 & 1.2 & 3.8 & 1.2 & 5.7 & 2.4 & 1.4 \\
Anode voltage, V & 150 & 300 & 150 & 150 & 300 & 300 & 500 \\
Xe ion energy, eV & 130 & 250 & 130 & 130 & 250 & 250 & 440 \\
Integrated sputter yield, atom/ion & 0.04 & 0.18 & 0.04 & 0.04 & 0.18 & 0.18 & 0.44 \\
Sputtered energy of carbon, eV & 1.4 & 2.1 & 1.4 & 1.4 & 2.1 & 2.1 & 2.9 \\
Momentum increase, \% & $\mathbf{0 . 1}$ & $\mathbf{0 . 4}$ & $\mathbf{0 . 1}$ & $\mathbf{0 . 1}$ & $\mathbf{0 . 4}$ & $\mathbf{0 . 4}$ & $\mathbf{1 . 0}$ \\
\hline
\end{tabular}

Because the sputter yield varies with incident particle angle, an integrated value was computed based on the probable incident ion trajectories from the Hall thruster channel impacting an annular section of the circular PMFS target. This is presented in Tables 4 and 5 as integrated sputter yield.

According to the SRIM simulations, the increase in measured momentum from carbon atoms sputtered off of the PMFS graphite target is at most $1.3 \%$ of the total measured momentum from the incident Xe ions. This small correction is included in all of the measurements presented in this paper, but is typically much less than the error associated with assuming azimuthal symmetry in the Hall thruster plume for the PMFS measurements.

\section{B. Neutral Reflection}

A small fraction of the particles from the P5 Hall thruster hit the PMFS target as cold, unaccelerated, neutrals. A cold neutral particle would bounce off the target, thus contributing twice the amount of momentum that it carries towards the PMFS target. However, these cold gas particles carry negligible momentum compared to the accelerated ions and in this case, no neutral gas corrections were needed. Furthermore, the fraction of unaccelerated neutrals to accelerated ions is approximately $20 \%{ }^{17}$ A more serious concern is hot neutrals created from fast ions undergoing charge exchange with the atoms of an ambient neutral gas population. Typically these energetic neutrals would go undetected by the Faraday probe that was used to detect the relative radial ion flux. However, as long as the charge exchange fraction is low compared to the ion population and/or the concentration of charge exchanged neutrals is proportional to the ion flux, charge exchanged neutrals do not significantly affect the accuracy of the PMFS device. As estimated by Randolph et al. ${ }^{14}$, only $2 \%$ of $200 \mathrm{eV}$ Xe ions undergo CEX within $100 \mathrm{~cm}$ with a background Xe pressure of $3 \times 10^{-6}$. With all of these factors combined, the cold gas contributes negligible momentum compared to the accelerated ions in the P5 Hall thruster at nominal operating conditions. 


\section{Conclusion}

The PMFS has proven to be considerably accurate compared to the inverted pendulum thrust stand with a maximum observed difference of approximately $6 \%$ and an average difference of approximately $2 \%$ over a large range of force, ion energy, and neutral gas flow settings. It is often cumbersome or impossible to mount heavy thrusters on pendulum-type thrust stands. Likewise, the cost associated with designing an appropriate thrust stand can be prohibitive to some research efforts. As a viable alternative, the PMFS can be used as a highly accurate force sensor for nearly all flowing plasma conditions. The PMFS may even allow full spacecraft-thruster integration testing, where a complete spacecraft, including thruster, could be assembled in a large vacuum chamber and tested as one integrated assembly. Because the PMFS has demonstrated its accuracy as a plasma force diagnostic and is a simple, robust, and low-cost device, it should find considerable application in the flowing plasma and electric propulsion communities.

\section{Acknowledgments}

The authors would like to thank the University of Houston Institute for Space Systems Operations (ISSO) postdoctoral fellowship program, and the Ad Astra Rocket Company for travel and experiment support.

\section{References}

${ }^{1}$ Chavers, D.G., Chang-Diaz, F.R., "Momentum Flux Measuring Instrument for Neutral and Charged Particle Flows," Rev. Sci. Instrum., Vol. 73, No. 10, 2002.

${ }^{2}$ Cohen, S.A. Zonca, F., Timberlake, J., Bennett, T., Cuthbertson, J., Langer, W., Motley, R., “An Instrument for Measuring the Momentum Flux From Atomic and Charged Particle Jets," Rev. Sci. Instrum. 61, 3586, 1990.

${ }^{3}$ Nedzelskiy, I.S., Silva, C., Fernandes, H., Duarte, P., Varandas, C.A.F, "Compact Cantilever Force Probe for Plasma Pressure Measurements," Rev. Sci. Instrum. 78, 123505, 2007.

${ }^{4}$ Yeha, F.B., Wei, P.S., "The Effect of Sheath on Plasma Momentum Transport to an Electrically Biased Surface," International Journal of Heat and Mass Transfer, Vol. 48, Issue 11, May 2005, pp. 2198-2208

${ }^{5}$ Haas, J.M., "Low-perturbation Interrogation of the Internal and Near-field Plasma Structure of a Hall Thruster Using a HighSpeed Probe Positioning System," Thesis, Dept. of Aerospace Engineering, University of Michigan, 2001.

${ }^{6}$ Haas, J.M., Gallimore, A.D., "An Investigation of Internal Ion Number Density and Electron Temperature Profiles in a Laboratory-Model Hall Thruster," AIAA Paper 2000-3422, July 2000.

${ }^{7}$ Haas, J.M. Gulczinski III, F.S., Gallimore , A.D., Spanjers, G.G., Spores, R.A, "Performance characteristics of a $5 \mathrm{~kW}$ laboratory Hall thruster," AIAA Paper 1998-3503,July 1998.

${ }^{8}$ Haas, J.M., and Gallimore, A.D., "Characterization of the Internal Plasma Structure of a 5 kW Hall Thruster," IEPC Paper 1999-078, Oct. 1999.

${ }^{9}$ Azziz, Y., "Experiemntal and Theorectical Characterization of a Hall Thruster Plume," Ph.D. Dissertation, Aeronautics and Astronautics Dept., Massachusetts Institute of Technology, Cambridge, MA, 2007.

${ }^{10}$ Ziegler, J.F., Biersack,J.P., SRIM: The Stopping and Range of Ions in Matter, Software Package, SRIM version 2008.

${ }^{11}$ Hofer, R.R., "Development and Characterization of High-Efficiency, High-Specific Impulse Xenon Hall Thrusters," Ph.D. Dissertation, University of Michigan, 2004.

${ }^{12}$ Haag, T.W., "Design of a Thrust Stand for High Power Electric Propulsion Devices," 25th AIAA/ASME/SAE/ASEE Joint Propulsion Conference, AIAA Paper 1989-2829, Jul. 1989.

${ }^{13}$ Gulczinksi, F.S., Hofer, R.R., and Gallimore A.D., "Near-field Ion Energy and Species Measurements of a $5 \mathrm{~kW}$ Laboratory Hall Thruster, " AIAA Paper 1999-2430, June 1999.

${ }^{14}$ Randolph, T., Kim, V., Kaufman, H. R., Kozubsky, K., Zhurin, V. V., Day, M. "Facility Effects on Stationary Plasma Thruster Testing," IEPC Paper 1993-093, Sept. 1993. 647.

${ }^{15}$ Stangeby, P.C., “The Plasma Boundary of Magnetic Fusion Devices," Instituute of Physics, Bristol, 2000, pp. 46, 113, and

${ }^{16}$ Doerner, R.P., Whyte, D.G., Goebel, D.M., "Sputtering Yield Measurements During Low Energy Xenon Plasma Bombardment,” J. Appl. Phys. 93, 5816, 2003.

${ }^{17}$ Hofer, R.R., Jankovsky, R.S., Gallimore, A.D., "High-Specific Impulse Hall Thrusters, Part 2: Efficiency Analysis," AIAA Journal of Propulsion and Power, 22, 4, 732-740, 2006. 